PROCEEDINGS OF THE

AMERICAN MATHEMATICAL SOCIETY

Volume 129, Number 9, Pages 2701-2707

S 0002-9939(01)06040-3

Article electronically published on February 9, 2001

\title{
A UNIQUENESS THEOREM WITH MOVING TARGETS WITHOUT COUNTING MULTIPLICITY
}

\author{
MIN RU
}

(Communicated by Steven R. Bell)

\begin{abstract}
In this paper, we prove a uniqueness theorem for holomorphic curves with moving targets without counting multiplicity.
\end{abstract}

\section{INTRODUCTION}

It is well known that two non-constant polynomials $f, g$ over an algebraic closed field of characteristic zero are identical if there exist two distinct values $a, b$ such that $f(x)=a \Leftrightarrow g(x)=a$ and $f(x)=b \Leftrightarrow g(x)=b$. In 1926, R. Nevanlinna (cf. [Ne) extended the above result to meromorphic functions. He proved that two non-constant meromorphic functions of a complex variable which attain five distinct values at the same points must be identical.

Wilhelm Stoll (cf. $[\mathrm{St}]$ ) later extended Nevanlinna's result to holomorphic curves and obtained the following theorem.

Theorem A1. Let $f_{1}, f_{2}, \ldots, f_{\lambda}: \mathbb{C} \rightarrow \mathbb{P}^{n}(\mathbb{C})$ be linearly non-degenerated holomorphic curves. Let $H_{1}, \ldots, H_{q}$ be hyperplanes in $\mathbb{P}^{n}(\mathbb{C})$ located in general position. Assume that $f_{1}^{-1}\left(H_{j}\right)=\cdots=f_{\lambda}^{-1}\left(H_{j}\right)$ and denote by $A_{j}=f_{1}^{-1}\left(H_{j}\right), 1 \leq j \leq q$. Assume further that for each $i \neq j, A_{i} \cap A_{j}=\emptyset$. Let $A=\bigcup_{j=1}^{q} A_{j}$. Let $l, 2 \leq l \leq \lambda$, be an integer such that for any increasing sequence $1 \leq j_{1}<j_{2}<\cdots<j_{l} \leq \lambda$, $f_{j_{1}}(z) \wedge \cdots \wedge f_{j_{l}}(z)=0$ for every point $z \in A$. If $q \geq \frac{\lambda n}{\lambda-l+1}+n+2$, then $f_{1}, \ldots, f_{\lambda}$ are algebraically dependent over $\mathbb{C}$, i.e., $f_{1} \wedge \cdots \wedge f_{\lambda} \equiv 0$ on $\mathbb{C}$.

In the case $\lambda=2$, Theorem A1 gives the following statement.

Theorem A2. Let $f, g: \mathbb{C} \rightarrow \mathbb{P}^{n}(\mathbb{C})$ be two linearly non-degenerated holomorphic curves. Let $H_{1}, \ldots, H_{3 n+2}$ be hyperplanes in $\mathbb{P}^{n}(\mathbb{C})$ located in general position. Assume that $f^{-1}\left(H_{j}\right)=g^{-1}\left(H_{j}\right), 1 \leq j \leq 3 n+2$, and for each $i \neq j, f^{-1}\left(H_{i}\right) \cap$ $f^{-1}\left(H_{j}\right)=\emptyset$. Let $A=\bigcup_{j=1}^{q} f^{-1}\left(H_{j}\right)$. If for every point $z \in A, f(z)=g(z)$, then $f \equiv g$.

This paper generalizes W. Stoll's result to moving targets. First, we introduce some notation. For a holomorphic curve $f: \mathbb{C} \rightarrow \mathbb{P}^{n}(\mathbb{C})$, we use $\mathbf{f}: \mathbb{C} \rightarrow \mathbb{C}^{n+1}-\{0\}$ to denote a reduced representation of $f$, that is $\mathbb{P}(\mathbf{f})=f$. We note that a hyperplane $H$ in $\mathbb{P}^{n}(\mathbb{C})$ can be regarded as a point in $\mathbb{P}^{n}\left(\mathbb{C}^{*}\right)$, where $\mathbb{C}^{*}$ is the dual space

Received by the editors January 18, 2000.

2000 Mathematics Subject Classification. Primary 32H30.

The author is supported in part by NSF grant DMS-9800361 and by NSA grant MDA904-991-0034. 
of $\mathbb{C}$. By a moving target we mean a holomorphic map $g: \mathbb{C} \rightarrow \mathbb{P}^{n}\left(\mathbb{C}^{*}\right)$. Let $\mathbf{g}: \mathbb{C} \rightarrow \mathbb{C}^{* n+1}-\{0\}$ be a reduced representation of $g$. Then $\mathbf{g}(\mathbf{f})$ is an entire function on $\mathbb{C}$. Note that although the function $\mathbf{g}(\mathbf{f})$ depends on the choice of representations, the zeros of $\mathbf{g}(\mathbf{f})$ however are independent of the the choice of representations. Moving targets $g_{j}: \mathbb{C} \rightarrow \mathbb{P}^{n}\left(\mathbb{C}^{*}\right), 1 \leq j \leq q$, are said to be in general position if there is $z_{0} \in \mathbb{C}$ such that the hyperplanes $g_{j}\left(z_{0}\right), 1 \leq j \leq q$, are located in general position. Let $f_{1}, f_{2}, \ldots, f_{\lambda}: \mathbb{C} \rightarrow \mathbb{P}^{n}(\mathbb{C})$ be non-constant holomorphic curves. Let $g_{j}: \mathbb{C} \rightarrow \mathbb{P}^{n}\left(\mathbb{C}^{*}\right), 1 \leq j \leq q$, be moving targets located in general position. Assume that $\mathbf{g}_{j}\left(\mathbf{f}_{t}\right) \not \equiv 0$ for $1 \leq j \leq q, 1 \leq t \leq \lambda$, and assume that $\left(\mathbf{g}_{j}\left(\mathbf{f}_{1}\right)\right)^{-1}\{0\}=\cdots=\left(\mathbf{g}_{j}\left(\mathbf{f}_{\lambda}\right)\right)^{-1}\{0\}$. Let $A_{j}=\left(\mathbf{g}_{j}\left(\mathbf{f}_{1}\right)\right)^{-1}\{0\}$. Denote by $T[n+1, q]$ the set of all injective maps from $\{1, \ldots, n+1\}$ to $\{1, \ldots, q\}$. For every $z \in$ $\mathbb{C}-\bigcup_{\beta \in T[n+1, q]}\left\{z \mid \mathbf{g}_{\beta(1)}(z) \wedge \cdots \wedge \mathbf{g}_{\beta(n+1)}(z)=0\right\}$, we define $\rho(z)=\#\left\{j \mid z \in A_{j}\right\}$. Then $\rho(z) \leq n$. For any positive number $r>0$, define $\rho(r)=\sup \{\rho(z)|| z \mid \leq r\}$ where the sup is taken over all $z \in \mathbb{C}-\bigcup_{\beta \in T[n+1, q]}\left\{z \mid \mathbf{g}_{\beta(1)}(z) \wedge \cdots \wedge \mathbf{g}_{\beta(n+1)}(z)=0\right\}$ with $|z| \leq r$. Then $\rho(r)$ is a decreasing function. Let

$$
d=\lim _{r \rightarrow \infty} \rho(r) \text {. }
$$

Then $d \leq n$. If for each $i \neq j, A_{i} \cap A_{j}=\emptyset$, then $d=1$. A number $r_{0}>1$ exists such that

$$
\rho(z) \leq d \leq n, \text { if }|z| \geq r_{0} \text { and } z \notin \bigcup_{\beta \in T[n+1, q]}\left\{z \mid \mathbf{g}_{\beta(1)}(z) \wedge \cdots \wedge \mathbf{g}_{\beta(n+1)}(z)=0\right\} \text {. }
$$

The following results are obtained in this paper.

Theorem 1. Let $f_{1}, f_{2}, \ldots, f_{\lambda}: \mathbb{C} \rightarrow \mathbb{P}^{n}(\mathbb{C})$ be non-constant holomorphic curves. Let $g_{j}: \mathbb{C} \rightarrow \mathbb{P}^{n}\left(\mathbb{C}^{*}\right)$ be moving targets located in general position and $T_{g_{j}}(r)=$ $o\left(\max _{1 \leq t \leq \lambda}\left\{T_{f_{t}}(r)\right\}\right), 1 \leq j \leq q$. Assume that $\mathbf{g}_{j}\left(\mathbf{f}_{t}\right) \not \equiv 0$ for $1 \leq j \leq q, 1 \leq t \leq \lambda$, and $\left(\mathbf{g}_{j}\left(\mathbf{f}_{1}\right)\right)^{-1}\{0\}=\cdots=\left(\mathbf{g}_{j}\left(\mathbf{f}_{\lambda}\right)\right)^{-1}\{0\}$. Denote by $A_{j}=\left(\mathbf{g}_{j}\left(\mathbf{f}_{1}\right)\right)^{-1}\{0\}$ and let $A=\bigcup_{j=1}^{q} A_{j}$. Let $l, 2 \leq l \leq \lambda$, be an integer such that for any increasing sequence $1 \leq j_{1}<j_{2}<\cdots<j_{l} \leq \lambda, \mathbf{f}_{j_{1}}(z) \wedge \cdots \wedge \mathbf{f}_{j_{l}}(z)=0$ for every point $z \in A$. If $q>\frac{d n^{2}(2 n+1) \lambda}{\lambda-l+1}$, then $f_{1}, \ldots, f_{\lambda}$ are algebraically dependent over $\mathbb{C}$, i.e, $\mathbf{f}_{1} \wedge \cdots \wedge \mathbf{f}_{\lambda} \equiv 0$ on $\mathbb{C}$.

When $n=1$ and $\lambda=2$, the above theorem yields the following result.

Corollary. Given two non-constant meromorphic functions $f, g$, assume that there exist seven meromorphic functions $a_{1}, \ldots, a_{7}$, with $a_{i} \not \equiv a_{j}$ for $i \neq j$, such that $T_{a_{j}}(r)=o\left(\max \left\{T_{f}(r), T_{g}(r)\right\}\right), 1 \leq j \leq 7$, and such that $f(z)=a_{j}(z) \Leftrightarrow g(z)=$ $a_{j}(z)$ for $1 \leq j \leq 7$. Then $f \equiv g$.

We note that Qinde Zhang [Z] had a better result of six small functions, instead of seven.

If we further assume that $f_{t}, 1 \leq t \leq \lambda$, are linearly non-degenerated, then we have a better result as follows.

Theorem 2. In addition to the assumptions in Theorem 1 we assume further that $f_{t}, 1 \leq t \leq \lambda$, are linearly non-degenerated. Then $f_{1}, \ldots, f_{\lambda}$ are algebraically dependent over $\mathbb{C}$ (in the sense that $\mathbf{f}_{1} \wedge \cdots \wedge \mathbf{f}_{\lambda} \equiv 0$ on $\mathbb{C}$ ) if $q>\frac{d n(n+2) \lambda}{\lambda-l+1}$. 


\section{Proof of Theorem 1 And Theorem 2} St].

We first recall some standard notation and definitions in Nevanlinna theory (cf.

Let $f=\left[f_{0}: \ldots: f_{n}\right]: \mathbb{C} \rightarrow \mathbb{P}^{n}(\mathbb{C})$ be a holomorphic map, where $f_{0}, \ldots, f_{n}$ are entire functions without common zeros. The Cartan characteristic function of $f$ is defined by

$$
T_{f}(r)=\int_{0}^{2 \pi} \log \max _{i}\left|f_{i}\left(r e^{i \theta}\right)\right| \frac{d \theta}{2 \pi}-\log \max _{i}\left|f_{i}(0)\right| .
$$

For holomorphic maps $f: \mathbb{C} \rightarrow \mathbb{P}^{n}(\mathbb{C}), g: \mathbb{C} \rightarrow \mathbb{P}^{n}\left(\mathbb{C}^{*}\right)$, let $n_{f, g}(r)$ be the number of zeros of $\mathbf{g}(\mathbf{f})$ in $|z|<r$, counting multiplicity, and let $n_{f, g}^{(n)}(r)$ be the number of zeros of $\mathbf{g}(\mathbf{f})$ in $|z|<r$, where the zero multiplicity $k$ is counted as usual if $k \leq n$ and the zero multiplicity is counted only as $n$ if $k>n$. The counting function is defined by

$$
N_{f, g}(r)=\int_{0}^{r} \frac{n_{f, g}(t)}{t} d t-n_{f, g}(0) \log r
$$

and the truncated counting function is

$$
N_{f, g}^{(n)}(r)=\int_{0}^{r} \frac{n_{f, g}^{(n)}(t)}{t} d t-n_{f, g}(0) \log r
$$

If $f$ is a meromorphic function, we use $n_{f}^{(n)}(r, 0)$ to denote the number of zeros of $f$ in $|z|<r$, where the zero multiplicity $k$ is counted as usual if $k \leq n$ and the zero multiplicity is counted only as $n$ if $k>n$. Let $N_{f}^{(n)}(r, 0)$ be the corresponding truncated counting function.

We recall the following Borel's lemma.

Theorem 2.1. Let $f=\left[f_{0}: \ldots: f_{n}\right]: \mathbb{C} \rightarrow \mathbb{P}^{n}(\mathbb{C})$ be a holomorphic map, with $f_{0}, \ldots, f_{n}$ entire and no common zeros. Assume that $f_{n+1}$ is a holomorphic function and $f_{0}+\cdots+f_{n}+f_{n+1}=0$. If $\sum_{i \in I} f_{i} \neq 0$ for any proper subset $I$ of $\{0, \ldots, n+1\}$, then

$$
T_{f}(r) \leq \sum_{j=0}^{n+1} N_{f_{j}}^{(n)}(r, 0)+O_{e x c}\left(\log r+\log ^{+} T_{f}(r)\right)
$$

for $r \rightarrow \infty$, where $O_{\text {exc }}$ means the estimate holds except for $r$ in a set of finite Lebesgue measure.

The proof of Theorem 2.1 is standard and can be found in [RW].

We then need the following "second main theorem type" inequality.

Theorem 2.2. Let $f: \mathbb{C} \rightarrow \mathbb{P}^{n}$ be a non-constant holomorphic map. Let $g_{j}$ : $\mathbb{C} \rightarrow \mathbb{P}^{n}\left(\mathbb{C}^{*}\right)$ be moving targets in general position. Assume that $\mathbf{g}_{j}(\mathbf{f}) \not \equiv 0$ for $j=1, \ldots, q$. If $q \geq 2 n+1$, then

$$
\frac{q}{n(2 n+1)} T_{f}(r) \leq \sum_{j=1}^{q} N_{f, g_{j}}^{(n)}(r)+O\left(\max _{1 \leq j \leq q} T_{g_{j}}(r)\right)+O_{e x c}\left(\log r+\log ^{+} T_{f}(r)\right) .
$$

The proof of Theorem 2.2 also appeared in [RW]. We include the proof here for the completeness. 
Proof of Theorem 2.2. Let $I \subset\{2, \ldots, q\}$ be the index set with the property that $i \in I$ if and only if

$$
T_{\frac{\mathbf{g}_{i}(\mathbf{f})}{\mathbf{g}_{1}(\mathbf{f})}}(r) \leq \sum_{j=1}^{q} N_{f, g_{j}}^{(n)}(r)+O\left(\max _{1 \leq j \leq q} T_{g_{j}}(r)\right)+O_{e x c}\left(\log r+\log ^{+} T_{f}(r)\right) .
$$

We first show that $\# I \geq n$. Assume that $I=\{2, \ldots, u\}$, and $u \leq n$. For dimension reason, $\left\{\mathbf{g}_{1}, \mathbf{g}_{n+1}, \ldots, \mathbf{g}_{2 n+1}\right\}$ is always linearly dependent over $\operatorname{Hol}(\mathbb{C})$, i.e.

$$
a_{1} \mathbf{g}_{1}+a_{n+1} \mathbf{g}_{n+1}+\cdots+a_{2 n+1} \mathbf{g}_{2 n+1}=0
$$

where $a_{1}, a_{n+1}, \ldots, a_{2 n+1}$ are holomorphic functions on $\mathbb{C}$. By solving the above linear equations for $a_{1}, a_{n+1}, \ldots, a_{n+2}$,

$$
T_{\left[a_{1}: a_{n+1}: \cdots: a_{2 n+1}\right]}(r) \leq 2(n+1)\left(\max _{1 \leq j \leq q} T_{g_{j}}(r)\right) .
$$

After rearranging the index we will have an equation

$$
a_{1} \mathbf{g}_{1}(\mathbf{f})+a_{n+1} \mathbf{g}_{n+1}(\mathbf{f})+\cdots+a_{m} \mathbf{g}_{m}(\mathbf{f}) \equiv 0
$$

and no proper subsum is identically zero as a function of $z$ where $m \geq n+1$; moreover we may assume without loss of generality that functions

$$
a_{1} \mathbf{g}_{1}(\mathbf{f}), \ldots, a_{m} \mathbf{g}_{m}(\mathbf{f})
$$

have no common zeros. Therefore, by Theorem 2.1, we conclude that

$$
T_{\frac{\mathbf{g}_{n+1}(\mathbf{f})}{\mathbf{g}_{1}(\mathbf{f})}}(r) \leq \sum_{j=1}^{q} N_{f, g_{j}}^{(n)}(r)+O\left(\max _{1 \leq j \leq q} T_{g_{j}}(r)\right)+O_{e x c}\left(\log r+\log ^{+} T_{f}(r)\right) .
$$

This contradicts the fact that $n+1$ is not in $I$. Thus $\# I \geq n$. So (2.1) implies

$$
\begin{aligned}
T_{f}(r) & \leq \sum_{j=2}^{n+1} T_{\frac{\mathbf{g}_{j}(\mathbf{f})}{\mathbf{g}_{1}(\mathbf{f})}}(r)+O\left(\max _{1 \leq j \leq q} T_{g_{j}}(r)\right) \\
& \leq \sum_{j=1}^{q} n N_{f, g_{j}}^{(n)}(r)+O\left(\max _{1 \leq j \leq q} T_{g_{j}}(r)\right)+O_{e x c}\left(\log r+\log ^{+} T_{f}(r)\right) .
\end{aligned}
$$

Denote by $\mathcal{L}_{q}$ the collection of moving hyperplanes $g_{1}, \ldots, g_{q}$ located in general position. We claim

$$
\frac{q}{2 n+1} T_{f}(r) \leq \sum_{g \in \mathcal{L}_{q}} n N_{f, g}^{(n)}(r)+O\left(\max _{g \in \mathcal{L}_{q}} T_{g}(r)\right)+O_{e x c}\left(\log r+\log ^{+} T_{f}(r)\right) .
$$

We will prove (2.3) by induction on $q$. When $q=2 n+1,(2.3)$ is just (2.2), so (2.3) holds. We assume (2.3) holds for $q$ and verify $(2.3)$ for $q+1$. In fact, for $\mathcal{L}_{q+1}$, we can choose $q$ moving hyperplanes at a time and apply (2.3). This gives $q+1$ inequalities as (2.3). Summing up these $q+1$ inequalities, we have

$$
\frac{q+1}{2 n+1} T_{f}(r) \leq \sum_{g \in \mathcal{L}_{q+1}} n N_{f, g}^{(n)}(r)+O\left(\max _{g \in \mathcal{L}_{q+1}} T_{g}(r)\right)+O_{e x c}\left(\log r+\log ^{+} T_{f}(r)\right) .
$$

This completes the proof of Theorem 2.2 . 
Proof of Theorem 1. We first apply Theorem 2.2 to $f_{t}, 1 \leq t \leq \lambda$, to get

$$
\frac{q}{n(2 n+1)} T_{f_{t}}(r) \leq \sum_{j=1}^{q} N_{f_{t}, g_{j}}^{(n)}(r)+O\left(\max _{1 \leq j \leq q} T_{g_{j}}(r)\right)+O_{e x c}\left(\log r+\log ^{+} T_{f_{t}}(r)\right) .
$$

Assume that $\mathbf{f}_{1} \wedge \cdots \wedge \mathbf{f}_{\lambda} \not \equiv 0$. We denote by $\mu_{\mathbf{f}_{1} \wedge \cdots \wedge \mathbf{f}_{\lambda}}$ the divisor associated with $\mathbf{f}_{1} \wedge \cdots \wedge \mathbf{f}_{\lambda}$. Denote by $N_{\mu_{\mathbf{f}_{1} \wedge \cdots \wedge \mathbf{f}_{\lambda}}}(r)$ the counting function associated with the divisor $\mu_{\mathbf{f}_{1} \wedge \cdots \wedge \mathbf{f}_{\lambda}}$. We make the following claim.

Claim. For every $1 \leq t \leq \lambda$ and $r \geq r_{0}$,

$$
\sum_{j=1}^{q} N_{f_{t}, g_{j}}^{(n)}(r) \leq \frac{d n}{\lambda-l+1} N_{\mu_{\mathbf{f}_{1} \wedge \cdots \wedge \mathbf{f}_{\lambda}}}(r)+\sum_{\beta} N_{\mu_{\mathbf{g}_{\beta(1)} \wedge \cdots \wedge \mathbf{g}_{\beta(n+1)}}}(r)
$$

where the sum is over all injective maps $\beta:\{1, \ldots, n+1\} \rightarrow\{1, \ldots, q\}$.

We now prove the claim. Let $z \in A$. Then for any increasing sequence $1 \leq j_{1}<$ $j_{2}<\cdots<j_{l} \leq \lambda$,

$$
\mathbf{f}_{j_{1}}(z) \wedge \cdots \wedge \mathbf{f}_{j_{l}}(z)=0 .
$$

We verify that $\mathbf{f}_{1} \wedge \cdots \wedge \mathbf{f}_{\lambda}$ vanishes at $z$ with the vanishing order at least $\lambda-l+1$. In fact, by the power series expansion for each component of $\mathbf{f}_{i}$, we can write, for $1 \leq i \leq \lambda$,

$$
\mathbf{f}_{i}(\zeta)=\mathbf{a}_{i}+(\zeta-z) \mathbf{h}_{i}(\zeta)
$$

where $\mathbf{a}_{i}$ is a constant vector and $\mathbf{h}_{i}(\zeta)$ is a holomorphic vector-valued function defined around $z$. Denote by $T[\alpha, \lambda]$ the set of all increasing injective maps from $\{1,2, \ldots, \alpha\}$ to $\{1,2, \ldots, \lambda\}$. For each $\eta \in T[\alpha, \lambda]$, there exists a unique $\hat{\eta} \in$ $T[\lambda-\alpha, \lambda]$ such that $(\operatorname{Im} \eta) \cap(\operatorname{Im} \hat{\eta})=\emptyset$. Abbreviate $\epsilon_{\eta}=\operatorname{sing} \eta$. (2.6) then implies that, for any $\eta \in T[l, \lambda]$,

$$
\mathbf{a}_{\eta(1)} \wedge \cdots \wedge \mathbf{a}_{\eta(l)}=0 .
$$

Thus,

$$
\begin{aligned}
\mathbf{f}_{1} \wedge \cdots \wedge \mathbf{f}_{\lambda} & =\sum_{\alpha=1}^{l-1}(\zeta-z)^{\lambda-\alpha} \sum_{\eta \in T[\alpha, \lambda]} \epsilon_{\eta}\left(\bigwedge_{j=1}^{\alpha} \mathbf{a}_{\eta(j)}\right) \wedge\left(\bigwedge_{k=1}^{\lambda-\alpha} \mathbf{h}_{\hat{\eta}(k)}\right) \\
& +(\zeta-z)^{\lambda} \mathbf{h}_{1} \wedge \cdots \wedge \mathbf{h}_{\lambda} .
\end{aligned}
$$

The lowest exponent of $(\zeta-z)$ is $\lambda-l+1$. This verifies that $\mathbf{f}_{1} \wedge \cdots \wedge \mathbf{f}_{\lambda}$ vanishes at $z$ with the vanishing order at least $\lambda-l+1$. This, together with the property of $\min \{m, n\} \leq n=\frac{n}{\lambda-l+1}(\lambda-l+1)$ and the definition of $d$, implies that

$$
\sum_{j=1}^{q} N_{f_{t}, g_{j}}^{(n)}(r) \leq \frac{d n}{\lambda-l+1} N_{\mu_{\mathbf{f}_{1} \wedge \cdots \wedge \mathbf{f}_{\lambda}}}(r)+\sum_{\beta} N_{\mu_{\mathbf{g}_{\beta(1)} \wedge \cdots \wedge \mathbf{g}_{\beta(n+1)}}}(r) .
$$

So the Claim is proved.

We now proceed with our proof. By the First Main Theorem of the exterior product (cf. [St], P.327),

$$
N_{\mu_{\mathbf{f}_{1} \wedge \cdots \wedge \mathbf{f}_{\lambda}}}(r) \leq \sum_{i=1}^{\lambda} T_{f_{i}}(r)+O(1) \text { and } N_{\mu_{\mathbf{g}_{\beta(0)} \wedge \cdots \wedge \mathbf{g}_{\beta(n)}}}(r) \leq \sum_{j=1}^{q} T_{g_{j}}(r) .
$$


Combining (2.5) and (2.7) yields

$$
\sum_{j=1}^{q} N_{f_{t}, g_{j}}^{(n)}(r) \leq \frac{d n}{\lambda-l+1} \sum_{i=1}^{\lambda} T_{f_{i}}(r)+O\left(\max _{1 \leq j \leq q} T_{g_{j}}(r)\right)+O(1) .
$$

This, together with (2.4), gives, for $1 \leq t \leq \lambda$,

$$
\begin{aligned}
\frac{q}{n(2 n+1)} T_{f_{t}}(r) \leq & \frac{d n}{\lambda-l+1} \sum_{i=1}^{\lambda} T_{f_{i}}(r)+O\left(\max _{1 \leq j \leq q} T_{g_{j}}(r)\right) \\
& +O_{e x c}\left(\log r+\log ^{+} T_{f_{t}}(r)\right) .
\end{aligned}
$$

Thus, by summing them up,

$$
\begin{aligned}
\frac{q}{n(2 n+1)} \sum_{t=1}^{\lambda} T_{f_{t}}(r) \leq & \frac{d n \lambda}{(\lambda-l+1)} \sum_{t=1}^{\lambda} T_{f_{t}}(r) \\
& +O\left(\max _{1 \leq j \leq q} T_{g_{j}}(r)\right)+O_{\text {exc }}\left(\log r+\sum_{t=1}^{\lambda} \log ^{+} T_{f_{t}}(r)\right),
\end{aligned}
$$

which gives a contradiction under the assumption that

$$
q>\frac{d n^{2}(2 n+1) \lambda}{\lambda-l+1}
$$

This completes the proof of Theorem 1 .

To prove Theorem 2, we need replace Theorem 2.2 with Theorem 2.3.

Theorem 2.3. Let $f: \mathbb{C} \rightarrow \mathbb{P}^{n}$ be a linearly non-degenerated holomorphic map. Let $g_{j}: \mathbb{C} \rightarrow \mathbb{P}^{n}\left(\mathbb{C}^{*}\right)$ be moving targets located in general position. If $q \geq n+2$, then

$$
\frac{q}{n+2} T_{f}(r) \leq \sum_{j=1}^{q} N_{f, g_{j}}^{(n)}(r)+O\left(\max _{1 \leq j \leq q} T_{g_{j}}(r)\right)+O_{e x c}\left(\log r+\log ^{+} T_{f}(r)\right) .
$$

Proof of Theorem 2.3. Denote by $\mathcal{L}_{q}$ the collection of moving hyperplanes $g_{1}, \ldots, g_{q}$ located in general position. We will prove (2.8) by induction on $q$. For any $n+2$ moving hyperplanes $g_{1}, \ldots, g_{n+2}$ located in general position, we have the following result due to S. Mori $[\mathrm{M}]$ :

$$
T_{f}(r) \leq \sum_{j=1}^{n+2} N_{f, g_{j}}^{(n)}(r)+O\left(\max _{1 \leq j \leq q} T_{g_{j}}(r)\right)+O_{e x c}\left(\log r+\log ^{+} T_{f}(r)\right) .
$$

We note that the method of proving (2.9) is to transform the moving hyperplanes to coordinate hyperplanes. For example, when $n=1$, the transformation is just like

$$
\frac{f-g_{1}}{f-g_{2}} \cdot \frac{g_{3}-g_{2}}{g_{3}-g_{1}}
$$

So (2.8) holds for $q=n+2$. We now assume (2.8) holds for $q$, and verify (2.8) for $q+1$. In fact, for $\mathcal{L}_{q+1}$, we can choose $q$ moving targets at a time and apply (2.8). This gives $q+1$ inequalities as (2.8). Summing up these $q+1$ inequalities, we have

$$
\frac{q+1}{n+2} T_{f}(r) \leq \sum_{g \in \mathcal{L}_{q+1}} N_{f, g}^{(n)}(r)+O\left(\max _{g \in \mathcal{L}_{q+1}} T_{g}(r)\right)+O_{e x c}\left(\log r+\log ^{+} T_{f}(r)\right) .
$$

This completes the proof of Theorem 2.3. 
Proof of Theorem 2. Assume that $\mathbf{f}_{1} \wedge \cdots \wedge \mathbf{f}_{\lambda} \not \equiv 0$. By the same method in the proof of Theorem 1, replacing Theorem 2.2 by Theorem 2.3, we can derive

$$
\begin{aligned}
\sum_{t=1}^{\lambda} T_{f_{t}}(r) \leq & \frac{d n(n+2) \lambda}{q(\lambda-l+1)} \sum_{t=1}^{\lambda} T_{f_{t}}(r)+O\left(\max _{1 \leq j \leq q} T_{g_{j}}(r)\right) \\
& +O_{e x c}\left(\log r+\sum_{t=1}^{\lambda} \log ^{+} T_{f_{t}}(r)\right),
\end{aligned}
$$

which gives a contradiction under the assumption that

$$
q>\frac{d n(n+2) \lambda}{\lambda-l+1} .
$$

This completes the proof of Theorem 2 .

\section{ACKNOWLEDGMENTS}

The author would like to express his gratitude to the referee for many helpful comments and suggestions.

\section{REFERENCES}

[F] Fujimoto, H.: The uniqueness problem of meromorphic maps into the complex projective space. Nogoya Math. J. 58, 1-23, 1975. MR 52:14395

[Ji] Ji, S.: Uniqueness problem without multiplicities in value distribution theory. Pacific J. of Math., 135, 323-348, 1988. MR 90b:32050

[M] Mori, S.: Remarks on holomorphic mappings. Comtempory Math., 25, 101-114, 1983. MR 85k:32045

[Ne] Nevanlinna, R.: Einige Eindeutigkeitssätze in der Theorie der meromorphen Funketionen. Acta. Math., 48, 367-391, 1926.

[RS] Ru, Min and Stoll, W.: The second main theorem for moving targets. J. Geometric Analysis, 1, 99-138, 1991. MR 92j:32098

[RW] Ru, Min and Wang, J.: A second main type inequality for holomorphic curves intersecting hyperplanes. To appear.

[S] Smiley, L.: Dependence theorems for meromorphic maps. Notre Dame Thesis, 1979.

[St] Stoll, W.: On the propagation of dependences. Pacific J. of Math., 139, 311-337, 1989. MR 91a:32037

[Z] Zhang, Q.:An uniqueness theorem for meromorphic functions with small growth functions. Acta Mathematica Sinica, 636, 827-833, 1993.

Department of Mathematics, University of Houston, Houston, Texas 77204

E-mail address: minru@math.uh.edu 